\title{
Analysis on Informatization Assistance in Deep Learning of College EFL Teaching*
}

\author{
Zhaoxi Wei \\ Beijing Information Technology College, Beijing, China \\ Gang Ji \\ Beijing Information Technology College, Beijing, China
}

\begin{abstract}
In the context of education informatization, it is imperative to realize deep learning in college EFL teaching. The connotation of deep learning coincides with that of critical thinking competency in college EFL teaching. Therefore, optimizing the learning support function, optimizing the structure of learning environment, stimulating topic-related resources interaction, optimizing the use of multiple assessment methods and optimizing the organic link between shallow learning and deep learning in terms of critical thinking competency will be conducive to the cultivation and development of critical thinking competency in college EFL teaching assisted by information technology and effective to the implementation of deep learning in EFL teaching.
\end{abstract}

Index Terms - deep learning, information technology, critical thinking competency, college EFL teaching

\section{INTRODUCTION}

Under the background of education informatization, the deep integration of information technology and education has become an important task to promote the education reform. Through the process of application, analysis, evaluation and creation, we can transform knowledge into skills, improve learners' ability to construct knowledge actively and solve real problems, and then carry out meaningful learning. Visibly, in the information age, learners' simple memory and understanding of knowledge can no longer satisfy people's desire to explore knowledge. Only when the learners have the ability of active searching, the deep information processing, the active knowledge construction, the critical high-level thinking, the effective application of knowledge transformation and transfer, and the solution of the problems, can they have the ability of active learning, lifelong learning and the ability of knowledge innovation, and can they realize the meaningful individualized construction of knowledge. Here, memory and understanding, application, analysis, evaluation and creation are not only learning styles, but also learning processes. Memory and understanding belong to the learning styles in the process of shallow learning, and belong to the level of low-level thinking. The application, analysis, evaluation and creation of the latter belong to the deep learning process, the process of learners' participation and thinking, and the high-level of thinking (He \& $\mathrm{Li}, 2005)$. The above-mentioned learning styles and learning processes are the application and practice of shallow learning and deep learning in the field of education and teaching, while the division of low-level thinking and high-level thinking is the understanding and expression of learning objectives from the perspective of psychological cognition. Therefore, concerning the way of learning, learning process and learning objectives, shallow learning and deep learning which are based on the teaching practice are an organic unity to promote the development of education and teaching. In order to understand the necessity of deep learning in the information age, the correlation between deep learning and EFL teaching, and how to promote the development and realization of deep learning by means of information technology, the following are discussed.

\section{DISCUSSION}

\section{A. Deep Learning and EFL Teaching}

The concept of deep learning originated from abroad. In the 1950s, Fairlance Marton and R Roger Saljo proposed two learning styles, shallow learning and deep learning. In 2005, Professor Li Jiahou of Shanghai Normal University summed up the typical characteristics of deep learning and put forward the concept of deep learning for the first time. Since then, a series of related academic research on deep learning has been carried out in China, from deep learning theory research, deep learning methods research to deep learning application research, and the general conclusions that have been reached are: (1) The deep learning is a learning process based on the progressive learning development of understanding, criticizing, transferring and association, which finally benefits learners to make decisions and solve problems. (2) The deep learning emphasizes information integration, promotes knowledge construction, advocates

\footnotetext{
* This paper is the phased and research-oriented achievement of established projects of Project of Beijing Information Technology College in 2016 (Project No. XY-YN-06-201618)
} 
heuristic, exploratory and participatory teaching, advocates active learning and lifelong learning; (3) The deep learning can improve the learning ability, reflect the initiative of learners, and make the high-quality and efficient group cooperation; (4) The deep learning does not exclude shallow learning, and they are separate continuum.

The above literature research and conclusion make the synthesized review on deep learning respectively from the interpretation of deep learning process, the progressive development of deep learning process, the effects of deep learning progress on learning ability, and the independent phrase but organic unity between shallow learning and deep learning during the learning. With the comprehensive research and understanding, deep learning helps to promote learners' active learning, emphasizes the relevance between knowledge and details, and stresses the understanding and construction of meaning. Deep learning is conducive to the cultivation and development of high-level thinking (application, analysis, evaluation and creation).

The cultivation of high-level thinking ability appears frequently in the educational documents and related reports of different levels in our country, which is similar to developing learners' innovative thinking and paying attention to the cultivation of students' ability to analyze and solve problems. The Outline of China's Medium and Long Term Education Reform and Development Program recently published in China also puts emphasis of education and teaching on the combination of learning and thinking; and it advocates heuristic, exploratory, discussing, and participatory teaching to help students learn to learn. It can be said that the concept of education and teaching in our country pays attention to cultivating college students' high-level thinking ability, which coincides with the elaboration of high-level cognitive skills in the field of psychological cognition and the goal of college EFL teaching. In the domain of Benjamin Bloom's Theory of Taxonomy, memorizing and understanding are designed as simple cognitive abilities according to the degree of difficulty in cognitive processes, while application, analysis, evaluation and creation as high-level cognitive skills. The latter is the core skill of fostering critical thinking competence in college EFL teaching.

At present, the literature investigation and its reviews on college EFL teaching and the cultivation of critical thinking competence can be concluded as follows: (1) The critical thinking competence in EFL is inseparable from college English language competence. In the framework of college English language competence, social competence (speculative competence) is incorporated with cognitive competence (language knowledge), functional competence (language skills) and strategic competence (learning strategies). The critical thinking competence has become an indispensable part of college English language proficiency, and it is also an important link. (2) The theory of fostering foreign language critical thinking doesn't have practical impact on the practice of college English curriculum. Theoretical research on the cultivation of college English critical thinking competency is fruitful, and a theoretical consensus on the role of foreign language curriculum in fostering critical thinking competence has reached from the theoretical point of view. The definition of critical thinking competence, the formulation of measuring the critical thinking competence and the macro-training strategies have been basically formed. However, the effectiveness of the strategies for fostering critical thinking competence based on the combination of language skills courses is lacking. (3) It is imperative to cultivate the critical thinking competence of college English curriculum with the help of information technology. The study on critical thinking competence of college English is more in traditional classroom, but fewer in technical applications. Network environment and information technology not only bring convenience to EFL teaching, but also provide many possibilities and opportunities for promoting critical thinking-oriented EFL teaching under the information technology environment. Language learners in the era of information bombardment, not only are flooded by information, but also are required to analyze, to judge and to process the large amount of information. Therefore, in EFL teaching, it is necessary to encourage students to find and solve problems by means of information technology, to collect data by logical thinking and to analyze and judge them, so as to promote the development of critical thinking competence and to prove its effectiveness by empirical methods.

Up to now, the development and cultivation of critical thinking competence in college English curriculum is not only to keep pace with the times, but also to promote the deep learning of college foreign language.

\section{B. EFL Teaching and Cultivation of Critical Thinking Competency}

A Summary of 20 Years' Studies on the Cultivation of College English Thinking Competence in China (1998-2017) (Wang, 2017) finds that: "For a long time, college English teaching has attached importance to the training of language competence in terms of knowledge structure and teaching methods, with emphasis on discourse analysis, long-difficult sentence patterns and explanation of difficult words." (p.94). Students' learning is also often confined to the memorization of lexical sentence patterns and the correct use of grammar. This kind of teaching one-sidedly emphasizes the mastery of language skills, but neglects the cultivation of students' critical thinking competency. Therefore, the learners' learning strategies of mechanical, passive learning and memorizing knowledge and details, as well as the teaching methods of repetitive practice and mechanical memory in foreign language education generally result in the shallow, inefficient and absence of critical thinking competency in college EFL teaching. At the same time, it is pointed out in Commenting on the Problems Existing in the Cultivation of Critical Thinking Competency in Foreign languages teaching in Colleges and Universities that foreign languages teaching should not over-emphasize the training of critical thinking competency while neglecting the cultivation of language-oriented ability, and foreign language curriculum in college should become an important carrier for the cultivation of critical thinking competency. At the same time, language-oriented ability of foreign language learning is the prerequisite to cultivate the critical thinking competency. Therefore, the development of foreign language proficiency must be based on the language-oriented knowledge of 
pronunciation, grammar and vocabulary. Meanwhile the cultivation of critical thinking competency should be based on language-oriented ability, but this does not mean that students with poor language proficiency can not develop critical thinking competency. In fact, any language material, as long as the teacher-carefully- designed material, could push the students' level of critical thinking to a higher level (Wen \& Sun, 2015). Research on the problems of fostering critical thinking competency in EFL teaching and their results enlighten us: (1) The premise of fostering critical thinking competency in college EFL teaching is clarified. Language skills are the prerequisite and basis for the development of critical thinking competency in college English curriculum. Therefore, language skills training is indispensable and cannot be crossed; (2) The carrier of fostering critical thinking competency in college EFL teaching is confirmed. College English curriculum is an important carrier for the cultivation and development of students' critical thinking competency. Therefore, relying on the content of college English curriculum, teachers who carefully design language materials and language activities can and will promote the development of learners' critical thinking competency. (3) It points out the guideline of fostering critical thinking competency in college EFL teaching. The shallow learning style and its related teaching strategies in college EFL teaching will only result in the absence of critical thinking competency. Both reasonable treatment of shallow learning style and deep learning style and organic integration of the two styles in the language skills training and critical thinking competency training can and will train and develop students to learn passionately, explore daringly and question courageously. (4) The individual equality of the cultivation of learners' critical thinking competency in EFL teaching is confirmed. The learners of college EFL teaching, regardless of their language proficiency, can and will improve their critical thinking competency through college English course.

From the above, it can be seen that emphasizing and carrying out the following principles are conducive to the cultivation and development of critical thinking competency in EFL teaching, and helpful to accomplish the educational goal of EFL teaching from shallow learning to deep learning. These guiding principles are individual equality in EFL teaching, the basic but solid function of language skills, the support of language content, the guarantee of language activity design and the organic combination of shallow learning and deep learning.

\section{ANALYSIS}

Concerning what have been discussed above, it should be noted here, that is, the auxiliary application of information technology in fostering critical thinking competency in deep learning of EFL teaching.

In the "Internet + " era, there is no absolute knowledge authority on the Internet. Everyone is not only the input of information, but also the disseminator and output of information. A large number of Internet applications and platforms provide communication and cooperation between different users, which makes the amount of Internet information increase geometrically. Therefore, if learners want to form independent opinion among massive information, they must have the ability of analysis, reasoning, judgment and selection. In order to gradually understand the truth and avoid misleading and to make fair and rational judgments, it is a realistic problem to cultivate the ability of critical thinking competency in EFL teaching.

Literature research shows that, mobile Internet technology is conducive to the development of critical thinking competency. Innovation of the blended learning mode which combines the traditional learning mode with the network learning form by flipping classroom, as well as optimization and utilization of resources and technical means which are from inside to outside of the classroom as well as from online to offline are to encourage students to locate problems, solve problems, to use logical thinking to collect data to analyze and judge, which can ultimately promote the development of critical thinking competency. For example, the rich information on the Internet gives learners the access to inquiry of learning, virtual learning community is constructed in cyberspace and visual learning is carried out with teaching video resources or software tools, or simulation exercises are carried out in virtual setting (Wen, 2017). However, literature research also shows that there still exist problems of the mixed learning mode that combines traditional learning with online learning, such as the lack of students' initiative while online learning, the failure to achieve the desired learning effect without effective supervision, etc.. The above problems leads to the occurrence of "breeding hotbed of shallow learning", which results from learners' passive performance and shallow level of communication in network learning space, such as looking through information or online chatting (Zhang, 2015).

Therefore, how to optimize the auxiliary effect of information technology on the deep learning of foreign languages, to effectively accomplish the deep learning of foreign languages assisted by information technology, and to cultivate and develop foreign language learners' critical thinking are of great importance. At present, literature research on deep learning in e-learning environment demonstrates from different perspectives the components of deep learning in promoting information technology environment: setting, interaction, experience and reflection (Zhang, 2014). Setting, which is the core and foundation, refers to the field of activity; interaction, experience and reflection are the key elements of knowledge construction, knowledge transfer and application, problem solving and creation. They interact with each other to promote learners' deep learning activities. As far as foreign language learners are concerned, the process of foreign language learning is a process in which learners construct knowledge, develop skills, enliven thinking, exhibit individuality and broaden their horizons under the guidance of teachers. Combined with the characteristics of EFL teaching and second language acquisition, the following optimization can be carried out:

A. Optimize the Learning Scaffolding Function of IT Means 
Under the learning environment involving students' activities, achieving the students' deep learning goal doesn't depend on the self-study in daily learning, but on the guidance and help from teachers in daily learning (Guo, 2016). Under the network environment, autonomous learning platform and learning software provide learners with opportunities and possibilities of autonomous learning in the form of in-class, out-of-class, on-line and off-line, To pay close attention to learners' learning process by means of information technology, to guide learners at any time, to answer questions at any time and to act as the necessary knowledge and emotion scaffolding for learners will reduce the risks and make up for shortcomings of individual differences in EFL teaching, deepen learners' cognition of language and things through cooperative interaction between teachers and learners, and ensure the effectiveness of learners' learning process in coordination, management and supervision.

\section{B. Optimize the Learning Environment of Meaning Construction as a Process by Means of IT}

The Dynamic Theory of Second Language Acquisition points out that learners are constantly approaching the ideal state of language acquisition in the cycle of input, interaction, output and feedback. The input of language is for the purpose of understanding the meaning, and the output of language is for the purpose of conveying meaning that can be understood by others. The acquisition of meaning is a process of construction, which is connected, contrasted and discussed with different texts, different sources of information and different people. The construction of meaning is a process of deep learning and a dynamic process. There is a need for participation and consultation. Therefore, the interactive platform and learning community created under the assistance of information technology provide infinite possibilities for students to share their views and communicate with each other, and provide students with the goal of knowledge construction and with the environment set-up. At the same time, communication and sharing between learners, through reading, thinking about the views of others and expressing their own thinking, enhance the learners' ability of language expression as well as their ability of thinking, judgment and evaluation. Therefore, learning based on comprehension, in-depth exploration, seeking meaning, learning to apply and reflective learning makes learners present deep level of cognitive expression, obtain positive learning emotion and develop effective learning interaction, which is the ultimate embodiment of deep learning.

\section{Optimize Interactions between Resources and Learners to Stimulate Their Interest by Means of IT}

In the era of network information bombardment, individual selection and individual interest greatly influence the screening of individual learning. At the same time, it also shows that textbooks are not the only way for students to obtain information. Under the massive network information, only choosing the topic-related resources that suit students' interests can promote effective interaction, active communication, and positive language learning. At the same time, the classroom learning involves the use of language and the social concern of the language use, instead of just staying at the level of language knowledge. It has demonstrated in the literature review that language is a social phenomenon. Language skill, at least speaking, is preferably acquired in interesting interactions with others. And the topic that arouses learners' interest and attention is of the utmost importance. Learners who are free to choose any material on the same subject in accordance with their own interests, concerning society, livelihood, studying and emotion etc., not only enhance the autonomy and enthusiasm of learning, satisfy their inquiring desires, but also fully mobilize their participation in learning, stimulate their curiosity and desire for cooperative communication, so that they will naturally and willingly pay attention to and make use of the correct language form to express the authentic meaning. The communication environment constructed between teachers and students and between students and students, which originates from interaction of topic-related resources, will encourage students to jump out of the confinement of textbook context and be free from the constraint of the subject or the topic. By combining interested topics and materials in daily life with the development of comprehensive language skills, such as listening, speaking, reading and writing, language learning habits of speculating, analyzing, discussing and evaluating are gradually cultivated and trained to foster learners' critical thinking.

\section{Optimize the Use of Multiple Assessment Means under IT}

The traditional evaluation means is test, which aims at the acquisition of students' knowledge, while the alternative evaluation means aims at the construction of knowledge. The former leads to shallow learning, while the latter leads to the use of deep learning methods. Therefore, learners' daily performance in foreign languages teaching, participation statistics, analysis reports and reflection reports, which belong to formative evaluation methods, are all shown in the form of visualization and data processing with the help of information technology to provide learners and teachers with the most direct and most immediate information for observation, feedback and reflection, and to play a role of information recording and interpretation of the evaluation of the deep learning process and its effect.

\section{E. Optimize the Organic Connection between Shallow Learning and Deep Learning by Means of IT}

Basing on learners' interest, we promote the interaction aiming at the construction of meaning. Setting the goal of developing learners' critical thinking competency, we promote the parallel development of language knowledge, comprehensive language skills and critical thinking. Thus, language activities are gradually extended from the shallow level practice of words and sentences, the practice of language skills to the meaning construction and internalization of language in the form of question-and-answer, dialogue and elaboration. For EFL teaching with emphasis on the 
development of language comprehensive skills, memory and understanding are regarded as the main learning strategies, repetition and mechanical memory as the main teaching methods, which keep foreign language learning staying at the shallow level of learning. But learners, with the help and guidance of their teachers, who are assisted by the network-based autonomous learning platform and learning software, spontaneously make the acquisition, comparison, judgment and evaluation of language learning, for example, whether the vocabulary, the sentence and the paragraph are appropriate or not, whether the discourse is logic or not. Such process of individualized speculation, discussion and questioning can not only improve the efficiency of learners' self-learning, promote deep learning, but also effectively foster their critical thinking on knowledge construction in foreign language learning.

\section{CONCLUSION}

Fisher (2008), an English scholar, points out that language teaching is facing a shift from the focus on language accuracy and fluency to the focus on critical understanding and expression of language. The shift of attention in language teaching coincides with the target interpretation of critical thinking competence of college foreign language and the connotation of deep learning in the field of education and teaching. What is imperative is to realize the deep learning in college EFL teaching, and what should be done now is to pay attention to the cultivation of critical thinking competency in college EFL teaching. Under the background of educational informatization, it is not only a move to keep pace with the times, but also an inevitable action to deal with the college foreign language reform in the information age, that is, to cultivate and develop the critical thinking competency of college foreign languages teaching by means of information technology and to achieve the goal of deep learning in college EFL teaching effectively.

\section{REFERENCES}

[1] Fisher. (2008). Teaching Comprehension and Critical Literacy: Investigation on Guided Reading in Three Primary Classroom. Literacy, 42(1), 19-28.

[2] Guo Hua. (2016). In-Depth Learning and Its Significance. Curricula, Textbooks, Teaching Methods, 36(11), 25-32.

[3] He Ling, Li Jiahou. (2005). Promoting In-Depth Learning for Students. Modern Teaching, 2005(5), 29-30.

[4] Wang Yingping. (2017). Summary of 20-year Research on Fostering Speculative Competence in Chinese College English (1998-2017). Overseas English, 2017(21), 94-95.

[5] Wen Qiufang, Sun Wen. (2015). Comments on the Problems Existing in the Cultivation of Dialectical Ability in Foreign Languages Teaching in Colleges and Universities. Theory and Practice of Foreign Languages Teaching, 2015(03), 6-12+94.

[6] Wen Xue. (2017). Review of Research on Depth Learning: Connotation, Teaching and Evaluation. Global Education Prospects, 46(11), 39-54.

[7] Zhang Qi. (2015). Relative Research on Self-Efficacy and Deep Learning under E-Learning Environment. E-Education Research, 36(04), 55-61.

[8] Zhang Qi. (2014). Review on Domestic Research into Deep Learning under E - learning Environment. Modern Distance Education, 2014(05), 60-67.

Zhaoxi Wei was born in Shanxi, China in 1975. She received her M.A. degree in Cross-Culture Studies from Beijing International Studies University, China in 2006.

She is currently an associate professor in Beijing Information Technology College, Beijing, China. Her research interests include cross-culture studies, informatization English teaching and research.

Prof. Wei is a member of the National Association of Foreign Language Education of China Education Society.

Gang Ji was born in Shanxi, China in 1974. He received his M.A. degree in Computer Applied Science from Tianjin University, China in 2006.

$\mathrm{He}$ is currently an engineer of software development and a research associate of Computer Technology Institute in Beijing Information Technology College, Beijing, China. His research interests include framework construction research as well as computer-assisted informatization teaching. 